\title{
Study of Deuteron with Modified Screen Coulomb (Yakawa) Potential
}

\author{
M. R. Shojaei \\ Department of physics, Shahrood University of Technology \\ P.O.Box 36155-316, Shahrood, Iran \\ A.A. Rajabi \\ Department of physics, Shahrood University of Technology \\ P.O.Box 36155-316, Shahrood, Iran \\ T. Karimi \\ Payam Noor University of Fariman, Iran \\ E-mail: M.R.Shojaei@shahroodut ac.ir
}

Received: April 30, 2010 Acceped: May 18, 2010 doi:10.5539/apr.v3n1p122

\begin{abstract}
In this paper the Schrödinger equation was solved with modified screen coulomb (yukawa) potential and the one pion exchange potential. A mathematical method has been presented. Analytical solution of the Schrödinger equation with modified yukawa potential has been calculated. Then the ground state energy of deuteron was determined. We have used the second potential as a perturbation potential and found the energy shift of grand state energy of deuteron. Then we have used numerical method and solving the Schrödinger equation numerically. Finally, the results were compared with Bonn group and the HQCD.
\end{abstract}

Keywords: Deuteron, Schrödinger equation, Modified yukawa potential

\section{Introduction}

One of the important fundamentals in nuclear physics is the suitable potential between nucleons. Considering the proper potential, we can calculate the static properties of nucleus and compare the result with experimental data (Bihar, et all., 1980). In nuclear physics, the potential may have any one of the three forms: Square wall, Gusian potential, Yukawa potential. These potentials are based on relative distance between proton and neutron (Giannini, et al., 2003). It depends only on distance between them (Chen, et al. 2007). Although the square well potential is easier to handle analytically, this potential is not accepted physical for nucleon - nucleon interaction. (Giannini, et al., 1990). In nature, even for short ranges, the potentials are not expected to be so sharply cut off. (Causal, et a.,1192). Yukawa potential is physically more plausible, because of its origin in the well- accepted meson exchange theory of Yukawa potential. They consist of a long range part which describes empirically the observed repulsion with a few parameters. (Dutt, et al., 1988). There are several reviews on the meson exchange approach to the NN interaction. But, then deuteron problem for such a potential cannot be solved analytically. Numerical and some approximate equivalent analytical methods have been developed to solve the deuteron problem with Yukawa and other potentials, with diffused edges (Yukawa 1935). To investigate nucleon- nucleon interaction, we selected, the simplest nucleus, deuteron. The deuteron problem has given us information about nuclear forces between neutron and proton. Scattering of neutrons and protons from deuterons, further has yielded information about the nucleon- nucleon interaction (Tegen et al. 1983). In the present work, we consider the modified Yukawa potential for central interaction of between proton and neutron and we expanded these potential for short rang to get the suitable potential. (Shojaei, et al., 2008). Then we present a mathematical method and solve exact analytical the Schrödinger equation (Rajabi, 2005) . We have suggested the method for solving the wave function, essentially based on an ansatz for the eigenfunctions. The method we have obtained in this article will be used for many kinds of interacting potentials between many-particle systems and can be used to improve many models in atomic and nuclear physics (Garavelli, et al.1991) (Yongyao, et al. 2006). In addition to the central potential, we must consider the other potential from the one meson exchange between 
nucleons. The real impetus to the concept of exchange forces was given by meson theory of nuclear forces, which has basic characteristics of exchange forces.

\section{Nucleon-Nucleon interaction and Schrödinger equation for two body systems}

In our recent work a new mathematical method is presented for solving the Schrödinger equation with two-body systems. We can write the nucleon- nucleon potential, as applicable to deuteron as (Hans, 2001)

$$
v=v_{c}(r)+v_{O P E}
$$

Here $V_{c}(r)$ is the central potential and $v_{\text {OPE }}$ is the one pion exchange potential. The central potential may have any one of the forms given as; Square well, Gaussian potential and Yukawa potential. In this section central potential is considered Yukawa potential as follow (Yukawa 1935)

$$
v_{c}(r)=-\frac{A}{r} \exp \left(-\frac{r}{R}\right)
$$

Where $l, R$ is constant. In the actual situation, the exchange pion, must give a characteristic spin and isospin dependence. Thus one can obtain spin - spin and tensor potential as follow (Hans, 2001)

$$
v_{\text {OPE }}=\left(v_{S S} \vec{\sigma}_{1} \cdot \vec{\sigma}_{2}+v_{T} S_{12}\right) \vec{\tau}_{1} \cdot \vec{\tau}_{2}
$$

Where $S_{12}=\left[3\left(\vec{\sigma}_{1} \cdot \hat{r}\right)\left(\vec{\sigma}_{2} \cdot \hat{r}\right)-\vec{\sigma}_{1} \cdot \vec{\sigma}_{2}\right]$ and $\vec{\sigma}_{1}, \vec{\sigma}_{2}$ are the pauli matrix. The isospin coefficient

$$
\begin{array}{lll}
\vec{\tau}_{1} \cdot \vec{\tau}_{2}=1 & \text { For } & I=1 \\
\vec{\tau}_{1} \cdot \vec{\tau}_{2}=-3 & \text { For } & I=0
\end{array}
$$

When spins of protons and neutrons are parallel to $\vec{r}$ then $S_{12}=2$ and the tensor force is attractive. When spins of protons and neutrons are perpendicular to $\vec{r}$ then $S_{12}=-1$ and the tensor force is repulsive. We have supposed the central part of the interaction $\left(v_{s S}, v_{T}\right)$ in Eq.(3) as follow:

$$
v_{s S}=\frac{1}{4 p}\left(\frac{g m}{2 m}\right)^{2} \frac{1}{r^{3}}
$$

This potential $V_{\text {OPE }}$ successfully explains NN phase - shifts at low energy, as well as the quadrupole deformation of the deuteron. In next section we solve the Schrödinger equation with modified yukawa potential.

\section{Bound state of deuteron}

In this section we have used the Schrödinger equation to calculation deuteron wave function with Yukawa potential. The two body problem may be reduced to a problem of a single particle of reduced mass $m$ moving in a central potential. The Schrödinger equation is with the N-N interaction is as following (Hans, 2001) (Chen, et al. 2005).

$$
-\frac{\hbar^{2}}{2 \mu}\left(\frac{\partial^{2} \phi_{I M S}}{\partial r^{2}}+\frac{2}{r} \frac{\partial \phi_{I M S}}{\partial r}\right)+\left(v_{c}(r)+v_{O P E}-\varepsilon+\frac{1(1+1)}{2 \mu r^{2}}\right) \phi_{I M S}=0
$$

Here 1 is the orbital angular momentum. For simplifying we consider $\hbar=c=1$. We can write the wave function as:

$$
\phi_{I M S}=\sum_{m_{s}}^{1, \Theta,-1} \frac{U(r)}{r} y_{\odot}^{\ominus} \chi_{1}^{m_{s}}
$$

Here $c_{1}^{m_{s}}$ is the spin part of wave function and the function $u(r)$ obeys the radial part of the Schrödinger equation as following: 


$$
\frac{d^{2} U(r)}{d r^{2}}=\left(-e-\frac{A}{r} e^{-\frac{r}{R}}+\frac{l(l+1)}{2 m r^{2}}\right) U(r)
$$

There are many methods for solving Eq.(8). For analytical solution of the radial Schrödinger equation we use modified Yuawa potential as follow:

$$
v(r)=-\frac{A}{r} e^{-\frac{r}{R}}=-\frac{A}{r}\left(1-\frac{r}{R}+\frac{r^{2}}{2 R^{2}}-\frac{r^{3}}{3 R^{3}}\right)
$$

In fig. 1 we have plotted the Yukawa potential and modified Yukawa potential. Comparing this plot we can conclude that the behavior of as the yukawa potential. There is no discernible difference between them. Then we can select this potential and solve the Schrödinger equation

$<$ Figure 1>

Substituting Eq. (9) in Eq. (8) we have:

$$
\frac{d^{2} U(r)}{d r^{2}}=\left(-e-\frac{A}{r}+\frac{A}{R}-\frac{A r}{2 R^{2}}+\frac{A r^{2}}{6 R^{3}}+\frac{l(l+1)}{2 m r^{2}}\right) U(r)
$$

In this paper we present a mathematical method for solving Eq. (10). To solve the difficulty of above equation, we suggest the following answer: (Shojaei, et al., 2007)

$$
u(r)=h(r) \exp [g(r)]
$$

From Eq. (11) we have:

$$
\frac{d^{2} u(r)}{d r^{2}}=\left[g^{\prime \prime}+g^{\prime 2}+\left(\frac{h^{\prime \prime}+2 g^{\prime} h^{\prime}}{h}\right)\right] u(r)
$$

Let us take $h(r)=1$ or ground state and $g(r)$ as:

$$
g(r)=-\frac{1}{2} \sigma r^{2}+\xi r+\delta \operatorname{Ln}(r)
$$

Eqs (12) and (10) yield $\sigma, \xi, \delta$ and the constraints between the potential parameters $\lambda$ and $R$. These read

$$
\sigma=\sqrt{\frac{\lambda}{6 R^{3}}} \quad \xi=-\frac{1}{4} \sqrt{\frac{6 \lambda}{R}} \quad \delta=l+1
$$

Having Eqs (13), (14) the ground state energy is as following:

$$
e=3 \sqrt{\frac{l}{6 R^{3}}}-\frac{3}{8} \frac{l}{R}
$$

Finally the wave function is as follow:

$$
f(r)=N r^{1} \exp \left(-\frac{1}{2} \sqrt{\frac{l}{6 R^{3}}} r^{2}-\frac{1}{4} \sqrt{\frac{6 l}{R}} r\right)
$$

Using Eq. (16), we can calculate the static properties of deuteron. In this paper we determine the ground state energy. In next section we have solved the Schrödinger equation numerically and find the ground state energy.

\section{Numerical method for solving Schrödinger equation with Yukawa potential}

Many different numerical methods have for solved Schrödinger equation. In this section we have calculated the ground state energy of deuteron with the Yukawa potential by using numerical method. One of the numerical methods is the Runge- kutta method. Runge-Kutta $4^{\text {th }}$ order method is a numerical technique used to solve 
ordinary differential equation. In this method we consider the boundary condition at the point $X_{1}$ then we used the step $h$ and find the wave function at any point. By far the most often used is the classical fourth-order Runge-Kutta formula, which has a certain sleekness of organization about it: (Press, 1992)

$$
\begin{gathered}
u_{n+1}=u_{n}+\frac{k_{1}}{6}+\frac{k_{2}}{3}+\frac{k_{3}}{3}+\frac{k_{4}}{6}+o\left(h^{5}\right) \\
x_{n+1}=x_{n}+h
\end{gathered}
$$

Where $k_{1}, k_{2}, k_{3}, k_{4}$ define as follow:

$$
\begin{aligned}
& k_{1}=u\left(x_{n}, y_{n}\right) h \\
& k_{2}=u\left(x_{n}+\frac{h}{2}, y_{n}+\frac{k_{1}}{2}\right) h \\
& k_{3}=u\left(x_{n}+\frac{h}{2}, y_{n}+\frac{k_{2}}{2}\right) h \\
& k_{4}=u\left(x_{n}+h, y_{n}+k_{3}\right) h
\end{aligned}
$$

Using Runge-Kutta $4^{\text {th }}$ order method we find the ground state wave function for yukawa potential .This wave function plotted the fig.2. In this paper we have selected $h=.001$ and $u_{\odot}=\odot$

$<$ Figure 2>

In Table .1 we have selected the best value for ground state energy of deuteron.

$<$ Table 1>

In addition Yukawa potential one of the important potential between nucleons is the one pion exchange potential. We consider this potential as a perturbation potential and calculated the energy shift of ground state of deuteron.

\section{The effect of the one pion exchange potential in ground state energy}

The one pion exchange potential plays a central role in any modern realistic description of the nucleon - nucleon interaction. According to perturbation theory, the first order shift to energy is the expectation value of perturbing potential. In the present paper, we can consider the one pion exchange as a perturbation potential (Hans, 2001)

$$
v_{\text {OPE }}=\left(v_{s S} \vec{\sigma}_{1} \cdot \vec{\sigma}_{2}+v_{T} s_{12}\right) \vec{\tau}_{1} \cdot \vec{\tau}_{2}
$$

Where

$$
v_{T}(r) S_{12}=\frac{D}{r^{3}}\left[3\left(\sigma_{1} \cdot \hat{r}\right)\left(\sigma_{2} \cdot \hat{r}\right)-\sigma_{1} \cdot \sigma_{2}\right]
$$

When spins of protons and neutrons are parallel to $\vec{r}$ then $S_{12}=2$ and the tensor force is attractive. When spins of protons and neutrons are perpendicular to $\vec{r}$ then $S_{12}=-1$ and the tensor force is repulsive. For deuteron we have $\vec{\sigma}_{1} \cdot \vec{\sigma}_{2}=1$ when the $\vec{\tau}_{1} \cdot \vec{\tau}_{2}=1 S_{12}=2$ the potential is as $v_{\text {OPE }}(r)=\frac{3 D}{r^{3}}$, when $\vec{\tau}_{1} \cdot \vec{\tau}_{2}=-3$, $S_{12}=2$ the potential is as $V_{\text {OPE }}(r)=-\frac{6 D}{r^{3}}$, the first order shift energy is the expectation value of perturbing potential.

$<$ Table 2>

The effect of one pion exchange potential due of the split of ground state energy of deuteron.

\section{Conclusion}

We have presented the mathematical method for solving the Schrödinger equation with modified yukawa potential .This method can be used for any potential and investigation of nucleons and two body system of nucleons. We can also study nucleus built of three nucleons. The method presented in this paper is general and is 
worth extending for the solution of other potentials.This method is very simple and useful in solving the other equations for example Dirac equation, Klein Gordon equation with central and noncentral potentials. Using of these methods we are able to calculate in a straight forward way all observable quantities.

\section{References}

Bihar S N, Hare A. (1980). Lett. Math. Phys. 4.

Causal R S, Parishes D. (1992). Phys. Lett A, 170.

Chen C Y, Dai T Q. (2007). Phys.Scr., 75.

Chen C Y, Dong S H. (2005). Phys.Lett.A, 335.

Dutt R, Khare A, Sukhatme U P. (1988). Am.J.Phys., 56:163-168.

Garavelli S L, Oliveira F A. (1991). Physical Review Letters, Vol, 66, No, 10.

Giannini M M. (1990). Rep. Prog. Phys., 54.

Giannini M M., Santopinto E, Vassallo A. (2003). Progress in Particle and Nuclear physics, 50.

Hans H S. (2001). Nuclear physics, experimental and theoretical, New Age International (2001).

Kim Y, Lee S. (2010). Nucl. Phys, A844 (2010).

Press W H, Teukolsky S A. (1992). Numerical Recipes in Fortran, University of Cambridge (1992).

Rajabi A A. (2005). Few-Body systems, 37,197-213.

Shojaei M R, Rajabi A A, Hassanabdi H. (2008). International Journal of Modern Physics, E, Vol, 17, No, 6.

Shojaei M.R, Rajabi A A. (2007). Iranian Journal of physics Research, Vol, 7, No, 2.

Tegen R., Schedl M, Weise W. (1983). Phys Lett, vol125.

Yongyao L ,.Xiangqian L, Kroger H. (2006). Sceince in china, Series G Physics, Vol, 49, No.1.

Yukawa M. (1935). Proc. Phys. Math. Soc., Jpn.17.

Table 1. Ground state energy of deuteron by various authors and experimental data

\begin{tabular}{|l|l|l|l|l|}
\hline Deuteron & $\begin{array}{l}\text { HQCD(Kim, et } \\
\text { al., 2010) }\end{array}$ & $\begin{array}{l}\text { Bonn B(Kim, et } \\
\text { al., 2010) }\end{array}$ & $\begin{array}{l}\text { Experimental } \\
\text { (Hans, 2001) }\end{array}$ & $\begin{array}{l}\text { Our Model } \\
\text { (Best value) }\end{array}$ \\
\hline Ground state energy & 2.3 & 2.224 & 2.22 & 2.228 \\
\hline
\end{tabular}

Table 2. Energy shift and binding energy of deuteron by using the perturbation potential

\begin{tabular}{|l|l|l|}
\hline & $t=1, S_{12}=2$ & $t=0, S_{12}=2$ \\
\hline Energy shift (KeV) & 12.519 & -25.039 \\
\hline $\begin{array}{l}\text { Ground state energy } \\
(\mathrm{MeV})\end{array}$ & 2.123 & 2.111 \\
\hline
\end{tabular}




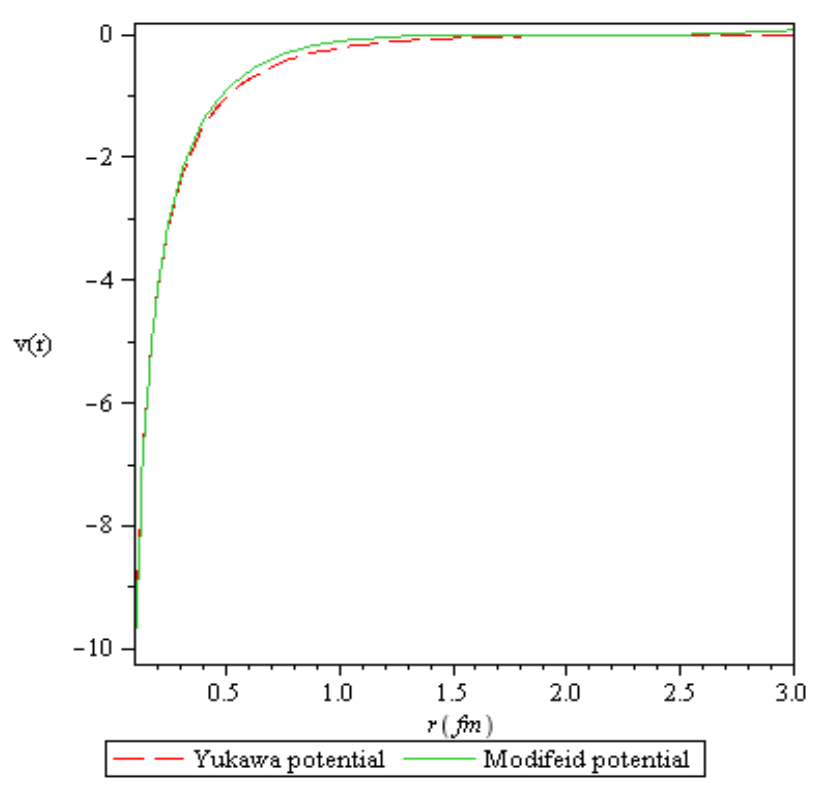

Figure 1. Comparison of the Yakawa potential with modified potential. The solid curve denoted the Yukawa potential , and the dashed curved denoted the modified potential

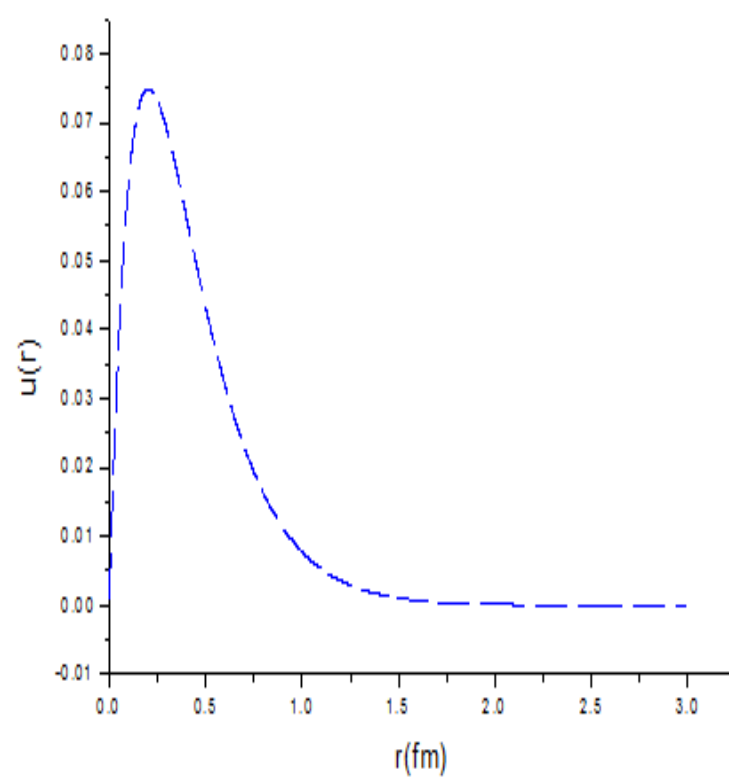

Figure 2. The ground state wave function for yukawa potential 